\title{
RECUPERAÇÃO DA FASE METÁlICA DAS PLACAS DE CIRCUITO IMPRESSO
}

\author{
N. M. MEDEIROS ${ }^{1}$, A. MICUSSI' ${ }^{2}$, D. A. dos $\operatorname{SANTOS}^{1}$, R. A. C. de MELO ${ }^{1}$, L. de F. \\ L. LUCENA ${ }^{3}$, C. P. de SOUZA ${ }^{1}$ \\ ${ }^{1}$ Universidade Federal do Rio Grande do Norte, Departamento de Engenharia Química \\ ${ }^{2}$ Universidade Federal do Rio Grande do Norte, Departamento de Engenharia Civil \\ ${ }^{3}$ Universidade Federal do Rio Grande do Norte, Escola de Ciências e Tecnologia \\ E-mail para contato: nataliamedeiros14@gmail.com; angelmicussi@yahoo.com.br; \\ da.vi.san@hotmail.com; raffael_melo_2@hotmail.com; 1llucena@yahoo.com.br; \\ carlson@ufrnet.br
}

\begin{abstract}
RESUMO - O consumo desenfreado de equipamentos eletrônicos juntamente com a rápida imersão de novas tecnologias no mercado impulsiona o crescimento acelerado de resíduos eletroeletrônicos. Esses resíduos sólidos por apresentarem muitos metais, dentre eles metais pesados, altamente tóxicos, que quando descartados de forma indevida, normalmente sem nenhum tratamento e junto com os demais resíduos urbanos, contaminam o meio ambiente e causam graves problemas a saúde humana. Há também metais preciosos e de base de alto valor agregado, que podem ser recuperados e reciclados, reduzindo a exploração de recursos naturais. Dessa forma, o objetivo deste trabalho consiste em estudar um processo que consiga separar a fração metálica das demais frações viabilizando o seu reaproveitamento. Os processos constituem em um tratamento físico e químico que juntamente com técnicas de caracterização, o material é estudado.
\end{abstract}

\section{INTRODUÇÃO}

É inegável o crescimento do consumo de produtos eletro/eletrônicos. Atrelado ao fato de que esses tipos de produtos têm um tempo de vida útil reduzido e que esse setor da indústria tem um desenvolvimento muito rápido, um considerável problema vem agregado a esses fatores - a produção de resíduo eletrônico (Veit, 2001; Kang e Schoenung, 2004).

A preocupação com o descarte correto desse tipo de resíduo se deve a quantidade de metais tóxicos presentes (Moraes, 2011), sendo geralmente manipulado e tratado de modo inadequado. Normalmente esses materiais, provenientes de equipamentos obsoletos e defeituosos, são empilhados com o lixo doméstico, jogados em aterros sanitários, queimados e podendo até serem deixados a céu aberto sem nenhum tratamento prévio (Veit, 2005; Zhang \& Forssberg, 1998).

O processo de reciclagem, quando realizado de forma inadequada, pode trazer problemas à saúde dos trabalhadores. Na China, esses efeitos negativos são bastantes elevados nas percentagens de $47,7 \%$ de dor de cabeça, $15 \%$ de coceira, $11,1 \%$ de náusea, 9,7\% de insônia, 5.3\% de amnésia e 4,8\% de conjuntivite (Qiu et al., 2005; Zhang, 2009). 
As placas de circuito impresso são encontradas na maioria dos equipamentos elétricos e eletrônicos (Marques et al., 2013) e os computadores apresentam a maior quantidade de PCI dentre os equipamentos elétricos e eletrônicos (Nnorom \& Osibanjo, 2008).

Junto com os seus componentes as PCI apresentam aproximadamente $40 \%$ de metais, $30 \%$ de plástico e $30 \%$ de cerâmica segundo He et al. (2006). Para as PCI sem os componentes $28 \%$ de fase metálica (FM) e $72 \%$ de fase não metálica (FNM) (Li et al., 2004).

Segundo Cui e Zhang (2008) os metais de alto valor podem ser recuperados do lixo eletrônico por processos piro-hidro e bio metalúrgicos. Porém, segundo Yang et al. (2011) os processos hidrometalúrgicos apresentam maior exatidão, são mais facilmente controlados e ambientalmente melhores do que os processos pirometalúrgicos.

Sendo assim, contemplando uma parcela desse amplo universo que é a problemática do lixo eletrônico, o presente trabalho tem como objetivo estudar e viabilizar uma rota relativamente simples para a recuperação da fração metálica presente nas PCIs de computadores desktops a partir de processos mecânicos e hidrometalúrgicos.

Esta pesquisa tem contribuição para a recuperação de alguns metais - que estão sendo estudados - tornando as PCIs uma fonte secundária de metais e atenuando seus impactos ao meio ambiente e assim, dando continuidade aos demais estudos que estão sendo realizados.

\section{METODOLOGIA}

O presente estudo caracteriza-se como uma pesquisa exploratória (qualitativa), com o objetivo de separar a fração metálica da fração polimérica e cerâmica presente em uma PCI retirada de computadores desktop obsoletos de marca desconhecida disponibilizado pelo Departamento de Materiais e Patrimônio (DMP) da Universidade Federal do Rio Grande do Norte (UFRN). Para isso a mesma foi submetida a uma série de processamentos - realizados em sua grande maioria no Laboratório de Termodinâmica do Departamento de Engenharia Química (DEQ) da UFRN.

\subsection{Processo Mecânico}

Essa etapa é necessária para o pré-tratamento do material. Procedimentos de desmontagem, corte, moagem e separação granulométrica são realizados para que se possa realizar o tratamento químico.

Inicialmente os capacitores, conectores, baterias e os demais componentes soldados a PCI foram retirados com ajuda de um soprador térmico, alicates e espátulas. Em seguida, após a desmontagem, a placa limpa foi cortada em pedaços menores de aproximadamente $4 \mathrm{~cm}^{2}$ em uma guilhotina tesourão no Laboratório de Oficina Mecânica do Departamento de Engenharia Mecânica (DEM) da UFRN com o intuito de facilitar a etapa seguinte de moagem. 
A fragmentação (cominuição) do material cortado foi realizada em um moinho de martelos como mostra a Figura 1 no Laboratório de Tratamento de Minérios da Diretoria Acadêmica de Recursos Naturais (DIAREN) do Instituto Federal de Educação, Ciência e Tecnologia do Rio Grande do Norte (IFRN).

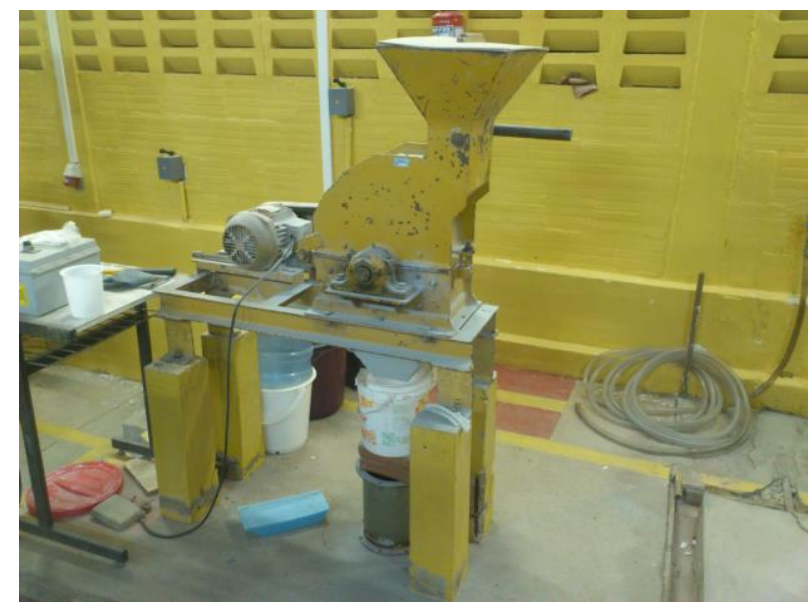

Figura 1 - Moinho de Martelos do IFRN.

Depois disso o pó formado foi recolhido e submetido a uma classificação granulométrica em uma peneira de 48 mesh $(0,3 \mathrm{~mm})$ agitada manualmente, dando origem assim a uma fração mais grosseira e outra mais fina.

\subsection{Processo Hidrometalúrgico}

A fração mais fina, do material fisicamente tratado, foi separada e submetida a uma lixiviação ácida em um erlenmeyer sob agitação de uma barra magnética, a temperatura ambiente e por 24 horas. Ainda, foram lixiviados $5 \mathrm{~g}$ do material caracterizado (passante na peneira de 48 mesh) em $100 \mathrm{ml}$ de um solução de ácido clorídrico e água (proporção 1:3) - além disso, cessada a agitação deixou-se a solução decantar por cerca de 42 horas.

Por último, com auxílio de um funil e papel de filtro, recolheu-se o corpo de chão da lixiviação. A amostra "A", como foi chamada, foi secada em um forno mufla a $60^{\circ} \mathrm{C}$ por 24 horas e caracterizada pelo método de Fluorescência de Raio X (FRX) - todas as caracterizações foram realizadas no Laboratório de Caracterização de Materiais do Departamento de Engenharia de Materiais (DCEM) da UFRN.

Foram executadas quatro precipitações consecutivas da solução lixiviada com uma solução de hidróxido de sódio $(\mathrm{NaOH}, 2$ molar) sob agitação constante e a temperatura ambiente, bem como filtrações, secagens e caracterização por FRX de cada precipitado formado ("A1", "A2", "A3" e "A4") - durante as precipitações o pH da solução foi constantemente aferido e registrados, através de papeis indicadores de $\mathrm{pH}$ e um pHmetro, bem como os volumes gastos de $\mathrm{NaOH}$.

\section{RESULTADOS E DISCUSSÕES}


Durante a precipitação pode-se observar variações macroscópicas na solução. Qualquer mudança nas características como cor, turbidez e floculação foi anotada.

A primeira mudança observada durante a precipitação foi quando a solução atingiu pH 4,30. A solução que estava límpida e verde ( $\mathrm{pH} 0$ ) conforme mostra Figura 2.a, apresentou uma coloração mais escura e de aspecto turvo como mostra Figura 2.b, indicando uma possível precipitação.



Figura 2: a) Solução de pH 0; b) Solução de pH 4,30 (Amostra A1).

A próxima alteração da solução foi registrada a um pH 8,43 em que apresentava uma coloração mais azulada como mostra Figura 3 e formação de flocos maiores conforme Figura 4 (precipitado).



Figura 3: Solução de pH 8,43 (Amostra A2). 


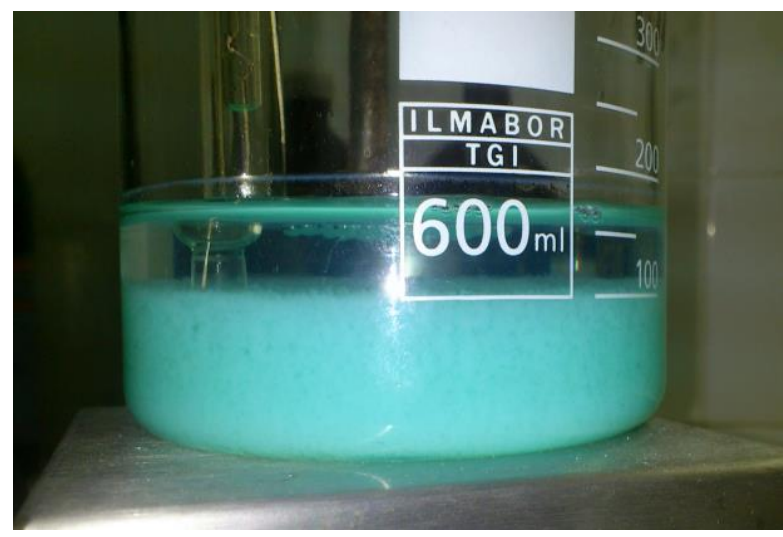

Figura 4: Formação de flocos da amostra A2.

As outras amostras A3 e A4 não apresentaram formação de precipitado em quantidade suficiente para serem analisadas. O resultado da análise do FRX das amostras A1 e A2 se encontram a seguir nas Figuras 5 e 6 respectivamente.

\begin{tabular}{|c|c|c|c|c|c|}
\hline Analyte & Result & \multicolumn{3}{|c|}{ Std.Dev. Proc.-Calc. Line } & Int. (cps/uA) \\
\hline $\mathrm{CuO}$ & $70.700 \%$ & $(0.058)$ & Ouan-FP & CuKa & 791.1420 \\
\hline $\mathrm{SnO2}$ & $14.845 \%$ & $(0.054)$ & Quan-FP & SnKa & 47.4636 \\
\hline $\mathrm{Fe} 203$ & $7.016 \%$ & $(0.022)$ & Quan-FP & FeKa & 56.1505 \\
\hline $\mathrm{S} 03$ & $4.401 \%$ & $(0.100)$ & Quan-FP & $\mathrm{S} \mathrm{Ka}$ & 0.2575 \\
\hline $\mathrm{PbO}$ & $1.643 \%$ & $(0.017)$ & Quan-FP & PbLb1 & 6.2756 \\
\hline $\mathrm{CaO}$ & $1.394 \%$ & $(0.014)$ & Quan-FP & CaKa & 0.5926 \\
\hline
\end{tabular}

Figura 5: FRX da amostra A1 de $\mathrm{pH} 4,30$.

Quantitative Result - Amostra A2

\begin{tabular}{|c|c|c|c|c|c|}
\hline Analyte & Result & \multicolumn{3}{|c|}{ Std.Dev. Proc.-Calc. Line } & Int. (cps/uA) \\
\hline CuO & $90.720 \%$ & $(0.065)$ & Ouan-FP & CuKa & 1333.5428 \\
\hline S03 & $5.253 \%$ & $(0.116)$ & Quan-FP & $\mathrm{S} \mathrm{Ka}$ & 0.3034 \\
\hline $\mathrm{PbO}$ & $3.748 \%$ & $(0.029)$ & Quan-FP & PbLb1 & 13.4925 \\
\hline $\mathrm{Ra}$ & $0.177 \%$ & $(0.009)$ & Quan-FP & RaLa & 0.9360 \\
\hline Th02 & $0.103 \%$ & $(0.006)$ & Quan-FP & ThLa & 0.8119 \\
\hline
\end{tabular}

Figura 6: FRX da amostra A2 de pH 8,43.

Na primeira amostra A1 a precipitação do cobre, na forma de óxido, foi muito maior do que os demais metais, atingindo $70,7 \%$. Isto também ocorreu para a segunda amostra em que a porcentagem de precipitação de cobre atingiu o valor de $90,7 \%$ e a diferença entre os demais metais foi ainda maior. 
Essa grande discrepância da precipitação do cobre em relação aos demais metais se deve ao fato, de o cobre ser o metal em maior quantidade nas placas de circuito impresso e, portanto, o mais fácil de ser removido, segundo Veit (2001).

Essas primeiras lixiviações são importantes para analisar a eficiência da remoção dos metais de base, principalmente o cobre que é o de maior concentração, uma vez que prejudica as lixiviações subsequentes dos próximos metais em menor concentração (Wang \& Gaustad, 2012; Birloaga et al., 2013). A recuperação do cobre, além de tudo, é também economicamente relevante.

A presença de metais radioativos como rádio ( $\mathrm{Ra}$ ) e tório $(\mathrm{Th})$ e de metais pesados como chumbo $(\mathrm{Pb})$ e estanho $(\mathrm{Sn})$ mostram a variedade de elementos metálicos dispostos nas placas de circuito impresso, evidenciando o poder de recuperação e reciclagem desse material.

Devido aos elevados custos de reciclagem e disposição adequada, a Coréia do Sul exporta aproximadamente 1,8 milhões de computadores usados para China a cada ano (Toxic Dispatch, 2004). Isso mostra a importância da implementação de um processo acessível para reciclagem e reaproveitamento dos REEE.

A reciclagem ajuda tanto no reaproveitamento dos materiais quanto ao meio ambiente. Segundo Robinson (2009), possibilita a recuperação de componentes reutilizáveis como também de materiais de base, porém devido às dificuldades tanto na complexidade do material quanto ao alto custo, muitos países não o fazem.

\section{CONSIDERAÇÕES FINAIS}

Através do desenvolvimento exploratório deste trabalho pode-se notar a viabilidade do processo adotado para recuperação de metais, em especial o cobre. Com a fragmentação do material (cominuição), com a homogeneização do pó pela separação granulométrica (peneiramento) e pelo tratamento químico da lixiviação já é possível uma recuperação significativa.

Isso é de grande importância para a situação atual e para os anos futuros onde a tendência ao consumo e ao descarte de equipamentos eletroeletrônicos ser cada vez mais crescente.

Um destino adequado e um processo de reaproveitamento economicamente mais acessível desses tipos de materiais são necessários, para que haja um maior investimento nessa área de reciclagem.

A viável recuperação desses metais das placas de circuito impresso torna-a uma ótima fonte de elementos metálicos, de grande aplicabilidade industrial, que são obtidos por processos físicos e químicos de baixo custo.

\section{REFERÊNCIAS}


BIRLOAGA, I.; DE MICHELIS, I.; FERELLA, F.; BUZATU, M.; VEGLIO, F. Study on the influence of various factors in the hydrometallurgical processing of waste printed circuit boards for copper and gold recovery. Waste Management, v. 33, p. 935-941, 2013.

CUI J. R.; ZHANG L. F. Metallurgical recovery of metals from electronic waste: a review. Journal of Hazardous Mater, v. 158, p. 228-56, 2008.

HE, W.; LI, G.; MA, X.; WANG, H.; HUANG, J.; HUANG, C. WEEE recovery strategies and the WEEE treatment status in China. J. Hazard. Mater., v. 136, p. 502$512,2006$.

KANG, H. Y., SCHOENUNG, J. M. Used consumer electronics: a comparative analysis of material recycling Technologies. International Symposium on Electronics and the Environment, Phoenix, Maio p. 10-13, 2004.

LI, J.; SHRIVASTAVA, P.; GAO, Z.; ZHANG, H. Printed circuit board recycling: a state of the art survey. IEEE Trans, Electron. Packag. Manuf., v. 27, p. 33-42, 2004.

MARQUES, A. C.; CABRERA, J. M.; MALFATTI, C. de F. Printed circuit boards: A review on the perspective of sustainability. Journal of Environmental Managemen, v. 131, p. 298-306, 2013.

MORAES, V. T. de, Recuperação de Metais a partir do Processamento Mecânico e Hidrometalúrgico de Placas de Circuito Impressos de Celulares Obsoletos, 2011, $120 \mathrm{f}$. Tese de Doutorado, Escola Politécnica, Universidade de São Paulo, São Paulo.

NNOROM, I. C.; OSIBANJO, O. Eletronic waste (e-waste): material flows and management practices in Nigeria. Waste Management, v. 28, p. 1472-1479, 2008.

PETTER, P. M. H., Avaliação da eficiência de lixiviação de metais preciosos das placas de circuito impresso com utilização de lixiviantes alternativos ao cianeto, 2012, 76f. Dissertação de Mestrado. Programa de Pós-Graduação em Engenharia de Minas, Metalúrgica e de Materiais, Universidade Federal do Rio Grande do Sul, Porto Alegre.

QIU, G.; FENG, X.; WANG, S.; SHANG, L. 2005 Mercury and methyl mercury in riparian soil, sediments, mine-waste calcines, and moss from abandoned $\mathrm{Hg}$ mines in east Guizhou province, southwestern China. Applied Geochemistry, v. 20, p. 627-638, 2005.

ROBINSON, B. H. E-waste: an assessment of global production and environment impacts. Science of theTotal Environment, v. 408, p. 183-191, 2009.

Toxic Dispatch. Environmentalists denounce toxic waste dumping in Asia. A newsletter from Toxic Links pp. 1-2 Toxic Dispatch n. 23, september, 2004.

VEIT, H. M., Emprego do Processamento Mecânico na Reciclagem de Sucatas de Placas de Circuito Impresso, 2001, 96f. Dissertação de Mestrado. Programa de Pós Graduação em Engenharia de Minas, Metalurgia e de Materiais, Universidade do Rio Grande do Sul, Porto Alegre. 
VEIT, H. M., Reciclagem de Cobre de Sucatas de Placas de Circuito Impresso, 2005, 101f. Tese de Doutorado. Programa de Pós Graduação em Engenharia de Minas, Metalurgia e de Materiais, Universidade do Rio Grande do Sul, Porto Alegre.

WANG, X.; GAUSTAD, G. Prioritizing material recovery for end-of-life printed circuit boards. Waste Management, v. 32, p. 1903-1913, 2012.

WIDMER, R.; OSWALD-KRAPF, H.; SINHA-KHETRIWAL, D; SCHNELLMANN, M.; BÖNI, H. Global perspectives on e-srcap. J. Environ. Impact Assess. Rev., v. 25, p. 436-458, 2005.

YANG, H.; LIU, J.; YANG, J.; Leaching copper from shredded particles of waste printed circuit boards. Waste Management, v. 32, p. 1903-1913, 2011.

ZHANG, S.; FORSSBERG, E. et al. Aluminum recovery from eletronic scrap by highforce ${ }^{\circledR}$ eddy-current separators. Resouces, Conservation and Recycling, v. 23, p. 225$241,1998$.

ZHANG, L.; From Guiyu to a nationwide policy: e-waste management in China. Environmental Politics, v. 18, p. 981-987, 2009. 